\title{
Automated Spacecraft Communications Service Demonstration Using NASA's SCaN Testbed
}

\author{
Dale J. Mortensen ${ }^{\mathrm{a}}$, Christopher Roberts ${ }^{\mathrm{b}}$, Richard C. Reinhart ${ }^{\mathrm{c}}$ \\ National Aeronautics and Space Administration, USA
}

\begin{abstract}
The traditional paradigm for space mission operations relies on inefficient, highly scripted pre-planned activities between space communications \& navigation service providers and user ground mission operations centers. Typically, there is limited or nonexistent automation capabilities on the user spacecraft for requesting space communications services, and on the provider network for request dispositioning and service provisioning. In the past, using these processes for space networks was sufficient with the relatively small number of user spacecraft requesting services. However, with an ever increasing number of satellites launched to orbit, more complex event-driven science objectives, exploration missions involving collaborative platforms, and more distant missions, approaches that improve automation, flexibility and efficiency are needed. This paper describes NASA's recently completed on-orbit demonstration of a new space communications service concept called User Initiated Service, its implementation, results, and a discussion for infusing this innovation into operations.
\end{abstract}

\section{Introduction}

A dvances in spacecraft capabilities and the simple increase in the number of spacecraft flying are calling for improvements to the supporting infrastructure. ${ }^{1,2}$ NASA currently operates three networks under the Space Communications and Navigation (SCaN) program. First, the Near Earth Network (NEN) allows earth orbiting spacecraft to communicate with ground stations as they pass within range. Second, the Space Network(SN), which is implemented with geostationary relay satellites, allows spacecraft more continuous communications as they circle the globe. The SN is also known as the Tracking Data Relay Satellite System or TDRSS. Thirdly, the Deep Space Network, with its very large aperture ground station antennas is used to communicate with planetary probes and similar assets. Demand will increase on all three of the current SCaN networks as the number of missions around earth and exploration missions beyond earth are expected to increase significantly in the future. Automation of the network services is one way to keep up with demand.

Traditionally, user missions begin the space communications operations planning process three to four weeks in advance of the service execution event. Users submit requests to access space link communications resources, which are dispositioned by the network scheduling office. Conflicts are adjudicated and resolved among scheduling and mission personnel. Finally, the pre-planned events are committed into a batch of operations, which are executed over the following week. Figure 1 illustrates this batch processing of request, disposition and pre-planned execution events in a rolling wave.

\footnotetext{
${ }^{a}$ Cognitive Communications Systems Lead, Communications and Intelligent Systems Division, NASA Glenn Research Center, Cleveland, Ohio, 44135, USA, AIAA non-member.

b Innovative Applications Mission Manager, Exploration and Space Communications Projects Division, NASA Goddard Space Flight Center, AIAA Member.

c Next Generation Relay Principal Investigator, Communications and Intelligent Systems Division, NASA Glenn Research Center, AIAA Member.
} 
Batch processing meets the needs of the relatively small number of user spacecraft serviced by the communications network, and for missions with regular or deterministic communications needs, such as steady-state science observations or launch operations. However, two related trends drive the need for a more responsive network service access protocol. First, more complex event-triggered mission operations cannot pre-plan the necessary communications services weeks in advance due to the stochastic nature of the scientific phenomena of interest. Second, the number of mission satellites is expected to dramatically increase as access to space increases and mission concepts evolve from large multifunctional monolithic platforms to many complimentary distributed platforms. ${ }^{3}$

In contrast to pre-planned batch processing, flow processing involves the timely execution of communication service events through a link channel. Figure 2 illustrates flow processing of a

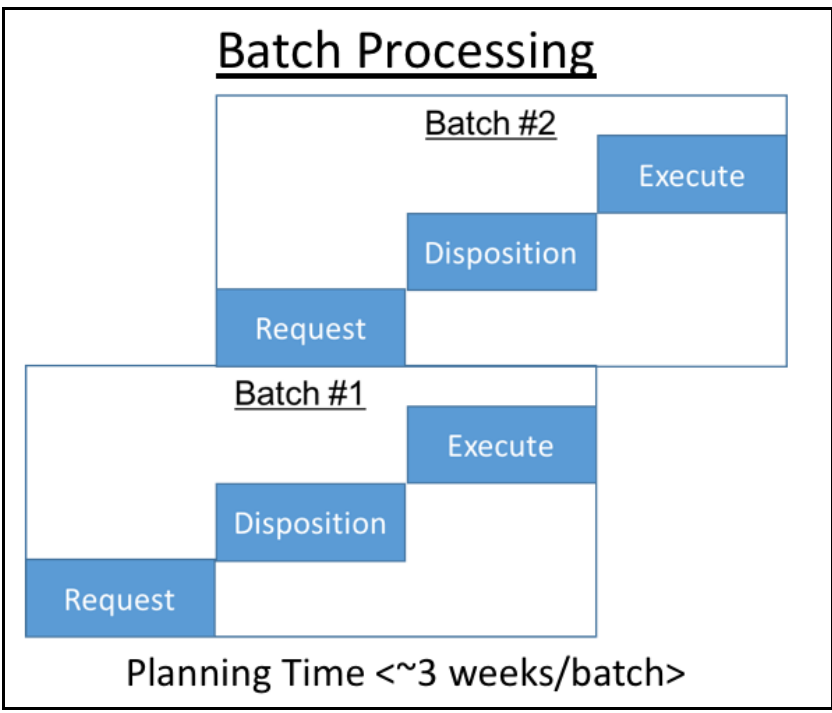

Figure 1. Traditional batch processing of space communications services communications event between a source and destination. Flow processing communications channels are prevalent in terrestrial wireless networks. Multiple simultaneous users are supported by sharing some aspect of the signal (e.g., space, frequency, bandwidth, or time), leading to higher availability at the expense of a lower effective data rate per user. Design concerns with flow processing for multiple access systems include characterizing the probabilistic demand loading, channel throughput and other considerations concerning blocking, queueing and traffic engineering. ${ }^{4}$

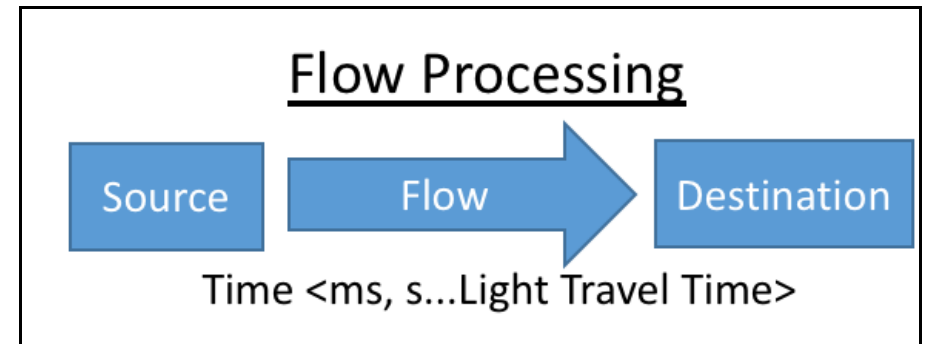

Figure 2. Illustrative flow processing of a communications event from source to destination
Space mission users have diverse needs and objectives. Three major drivers in space communications design and operations planning are user data volume, data delivery latency tolerance, and predictability of service demand. Constraints include spectrum policy, link channel access, availability, and other orbital or signal phenomena. ${ }^{5}$ Single access space link resources using high-gain terminals on geosynchronous relays or at ground stations can generally support higher data rates (at a given communications band) than multiple access link resources. Since a single

access resource typically involves pointing narrow field-of-view terminal towards the mission spacecraft and user specific equipment configurations, the use of batch processing allocates this terminal resource to users one at a time, with setup and teardown of configuration times on the order of a few minutes between user service events.

A new service access protocol, known as User Initiated Services (UIS), involves the automated use of narrowband multi-access link resources to request service from wideband single access link resources. ${ }^{6,7}$ This protocol enables significant improvements in user service request disposition and service event execution response times. Figure 3 illustrates the improved responsiveness UIS provides through the use of narrowband on-demand link channels to create on-the-fly batches for access to wideband resources. In the limit that the wideband resource has instantaneous setup and teardown configuration times it effectively becomes a flow resource. Based on experimental results presented in this paper, on-the-fly batch times on the order of tens of minutes are achievable with NASA's currently operational SCaN link resources. 
In communications networks, a network management architectural principle known as separation of concerns defines two functional blocks for information exchanges, a control plane and a data plane. ${ }^{8}$ Network controlrelated information exchanges are considered to take place within the control plane, and user information transfers occur in the data plane. Both planes can function over the same physical link, if needed. For the UIS, all users send service requests (via on-demand link) and receive network responses (e.g. broadcast link) through a dedicated control signaling plane that supports multiple access flow processing. Requested services are typically provided through single access, higher rate links, considered to be part of the data plane. Figure 4 illustrates this architecture which includes the satellite relay and user spacecraft, along with the central infrastructure node (i.e Event Manager, described later) working with the mission operation centers on the ground. Please note that in Figure 4 and other sections of the paper the control plane in the forward direction is depicted as a broadcast service, but this is not the only possible UIS implementation.

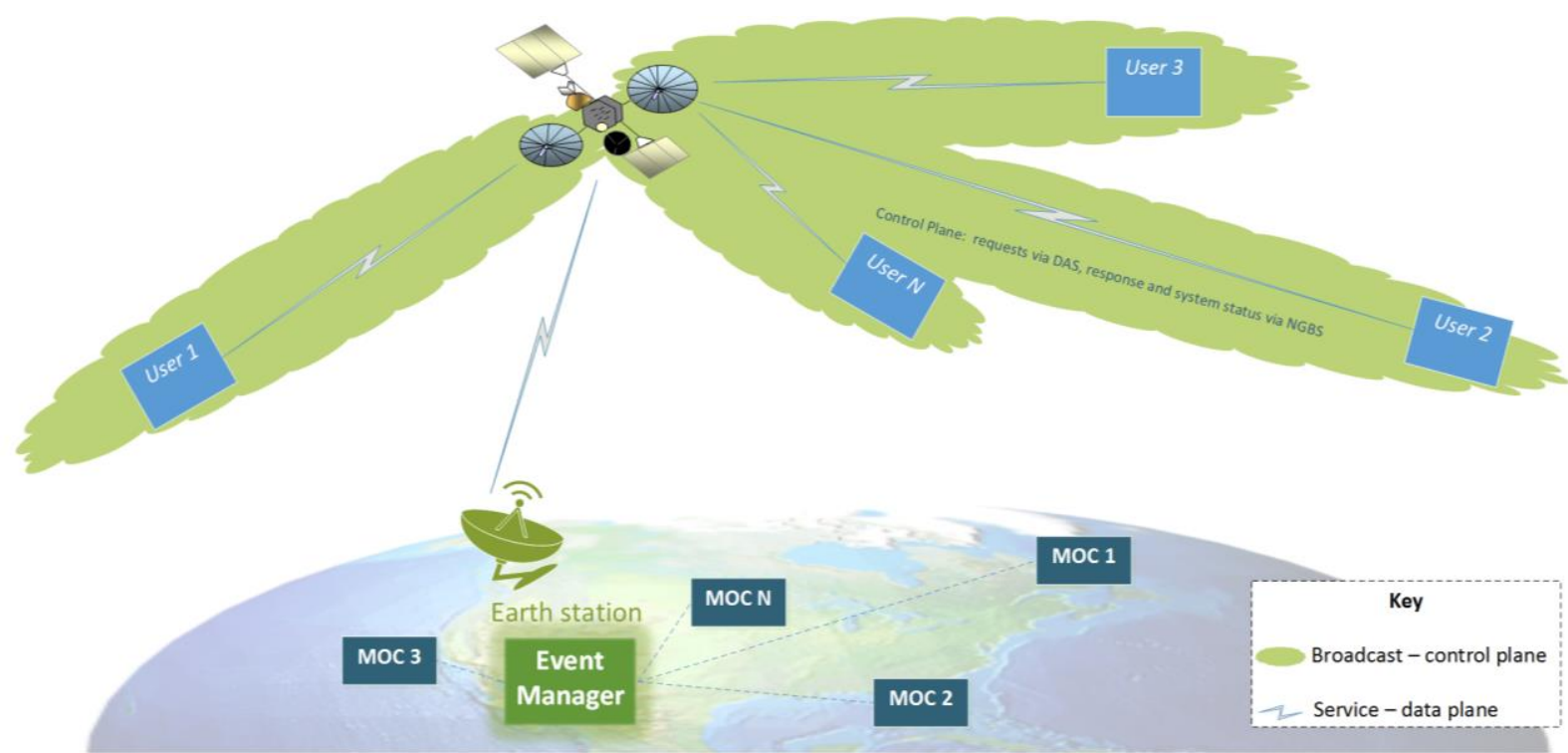

Figure 4. UIS Control \& Data Plane Concept

The remainder of the paper proceeds as follows. Section II discusses the messaging and protocols to support UIS, and describes the implementation used in the demonstration. Section III defines the demonstration system's pertinent components (e.g. Event Manager, Orbit Propagator, and Service Manager), followed by the experiment results in Section IV. Plans for additional on-orbit experiments, future infusion to regular operations, and use of commercial services are all in the Future Plans, Section V.

American Institute of Aeronautics and Astronautics 


\section{User Initiated Service Messaging and Protocols}

The basic messaging requirements to enable UIS functionality are a request mechanism to the network, and a corresponding response back to the user with access information. In general, the user request contains the information about the requested service type, priority, quantity of data to downlink and other parameters. Likewise, the response from the network contains response type (e.g. simple acknowledgement or other) and network access information if available. There are a few obvious service types, as shown in Table 1, but more certainly could be added to the protocol as needed. The message protocol distinguishes between requests and responses, as well as the various types of each.

With this protocol in place, a typical exchange between the user spacecraft and the ground Event Manager is illustrated in Figure 5. Notice the user first subscribes with the Event Manager, to initialize and maintain state, allowing service request processing. Similarly when all needed service has been acquired the user unsubscribes from the network, thus freeing Event Manager processing for other users.

For the on-orbit demonstration the experiment implemented a draft UIS over-the-air protocol defning a message header and data as shown in Figure 6. This defines a fixed message header with UIS specific parameters in addition to checksums on the header and message data for validation at the destination. TCP/IP encapsulation is used to send and receive UIS messages with the control plane links, as depicted in Figure 7.
Table 1. Service Types

\begin{tabular}{|c|c|c|}
\hline Type & Name & Application \\
\hline 0 & Subscription/Registration & $\begin{array}{l}\text { Registration and Time } \\
\text { Synchronization }\end{array}$ \\
\hline 1 & Data Volume & Science Data Downlink \\
\hline 2 & Open Downlink Channel & Telemetry \\
\hline 3 & Open Uplink Channel & Command \\
\hline 4 & $\begin{array}{l}\text { Radiometric Tracking } \\
\text { Service }\end{array}$ & Navigation \\
\hline 5 & $\begin{array}{l}\text { Optometric Tracking } \\
\text { Service }\end{array}$ & Navigation \\
\hline 6 & Emergency & $\begin{array}{l}\text { Mission specific e.g. high } \\
\text { temp, low battery }\end{array}$ \\
\hline
\end{tabular}

Ground

Event

Manager
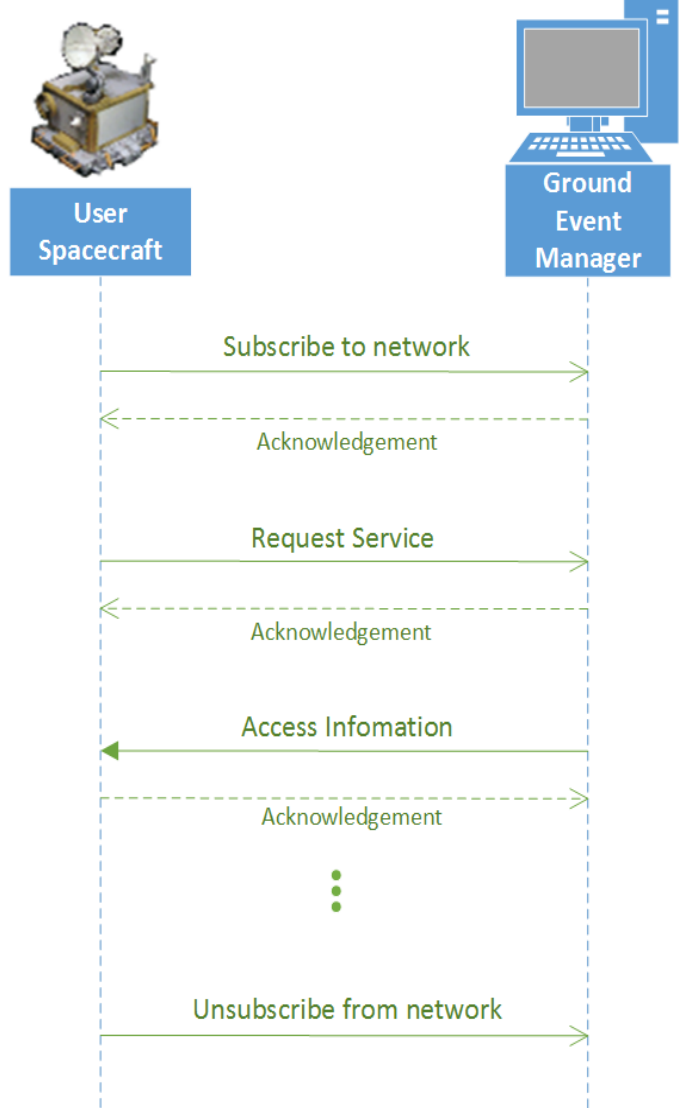

Figure 5. Typical UIS Message Sequence

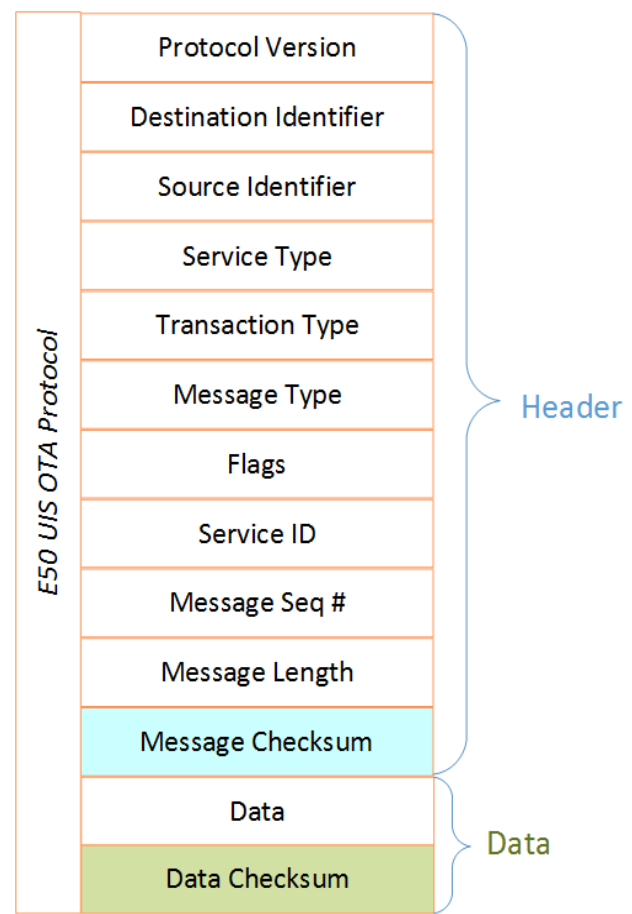

Figure 6. UIS Message Format 


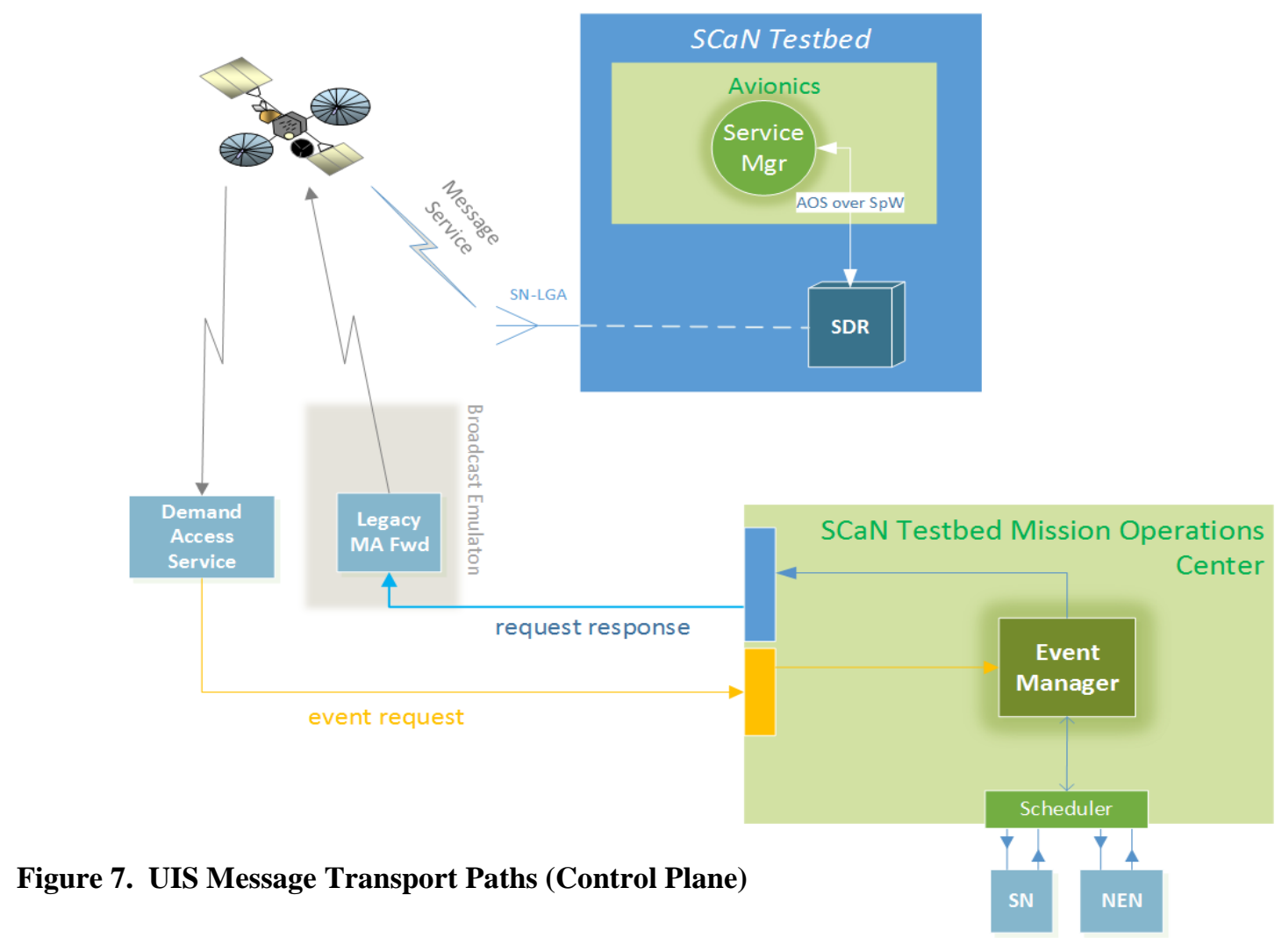

III. Experiment Systems, Components, and Operations

The Space Communications and Navigation (SCaN) Testbed is well suited for UIS and other experimental space communication demonstrations. The flight system is fully reconfigurable and allows adaptive interaction with ground systems. Launched in 2012 this external International Space Station (ISS) payload has three software defined radios (SDRs) and multiple antennas for establishing links directly with the ground or other orbiting spacecraft. ${ }^{9}$ Because the radios are software defined their functionality can be changed on the fly for different experiments by uploading new software. ${ }^{10}$ Also important is that the SCaN Testbed's controlling Avionics computer has an experiment application programmers interface (API), allowing users to upload and execute custom experiment software. Figure 8 is a graphic of the SCaN Testbed flight payload, where the external antennas, fixed and steerable are easily seen. The enclosure houses the SDRs, Avionics computer, and other supporting subsystems.

Since the SCaN Testbed is mounted on the ISS external truss there are antenna views of NASA's SN TDRSS relays in geostationary orbit as well as views of ground stations over which the ISS orbits. The experiment makes significant use of the SN relays for UIS control plane functions. This demonstration specifically uses the SN's demand access service (DAS) for the service request functionality because DAS can be always listening, an ideal UIS control plane characteristic. SCaN Testbed's fixed zenith facing antenna establishes this control plane link.

For data plane service connectivity, the SCaN Testbed establishes links with an S-band ground station located at NASA's Glenn Research Center (GRC), as well as relay links with TDRSS. For the desired higher data rate service links, SCaN Testbed's higher gain steerable antennas are employed for links with TDRSS. The Testbed uses a fixed nadir facing antenna for links with the GRC ground station. ${ }^{11}$ 


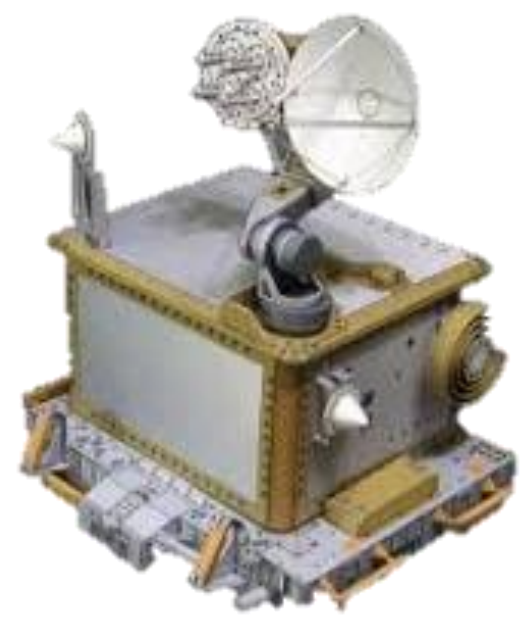

Figure 8. SCaN Testbed Flight Payload

The team developed and integrated several new pieces of software for both the flight and ground systems enabling the UIS experiment for the SCaN Testbed. First, an autonomous on-board antenna pointing system, includes an efficient orbital propagator such that SCaN Testbed knows to which TDRS and when to point the data plane link antenna. The propagator then tracks and maintains the scheduled service link. Second, a new waveform, implemented on the SDR works with the SN's DAS in a very efficient manner, allowing maximum access for making requests and minimizing radio power consumption. Third, an autonomous service manager operates on the flight avionics, initiating requests, processing service window responses, sending information to the antenna pointing system, and configuring the SDRs appropriately. Fourth, an event manager on the ground receives incoming requests from users and queries the $\mathrm{SCaN}$ Networks for available time slots. These four main software components will be described in more detail in the following subsections.

\section{A. Orbit Propagator}

By their very nature, orbits for most satellites, natural or artificial, are predictable within some degree of accuracy. There are several ways to compute an orbital prediction, however, the limited processing capacity and memory resources onboard a typical spacecraft requires this be done most efficiently. Previously, all SCaN Testbed experiment operations used an uploaded track file created from ground calculations dictating each pointing step for the gimbaled antenna. For UIS experiments that track file is effectively created on-board SCaN Testbed in the Avionics as needed for a granted service request. This on-board capability allows relatively small uploads of access information for granted services, such as a Ka-band access with TDRSS, thus reducing the necessary control plane bandwidth.

\section{B. Demand Access Service(DAS) Waveform}

NASA's Space Network DAS provides continuous and automated communications links via the TDRSS multiple access return capabilities. The service is enabled with the multiple access beamformer subsystem, which allows the SN to effectively track multiple users simultaneously. The DAS receivers work with the programmable telemetry processor, allowing users to retrieve downlinked data via the Internet. ${ }^{12}$ For the UIS SCaN Testbed DAS waveform implementation several configurable parameters were specifically chosen. First, the lowest DAS signaling rate of 2 kilo samples-per-second was chosen with the goal of maximizing link margin and access time during the ISS orbit. Since a fixed antenna is used for the DAS link, to leave the steerable antennas free for data downlinks, lowering the DAS rate allows the link to work significantly off-boresight while transmitting to a TDRS. Secondly, the time to transmit the UIS request is minimized to about 15 seconds. This is close to the minimum time the DAS receivers need to synchronize on the spread-spectrum BPSK signal. The waveform uses a sequence of idle frames before the 260 byte UIS request message is sent near the end of the 15 second transmission.

\section{Service Manager}

Once started, the on-board Service Manager monitors the spacecraft systems to determine when and how often service is requested from the network. If a data downlink or maintenance window is needed, then an UIS request is sent via the DAS Waveform. In the SCaN Testbed demonstration, the Service Manager responds to a buffer of data exceeding a configurable threshold. Upon receiving a response to the UIS request from the network the Service Manager configures the antenna system and appropriate SDR for the granted access. During an access event the Service Manager orchestrates the science data transfer from storage buffers to the downlinking SDR, or whatever data transfer needs to occur, downlink and/or uplink.

\section{Event Manager}

On the ground an Event Manager receives requests from network user spacecraft and works with the Space Network Access System (SNAS) to schedule appropriate service. It works with the real-time period as opposed to the forecast-period scheduling. For the SCaN Testbed experiment, unscheduled and available TDRSS time was requested, for which there is typically access events available within an orbit, depending on the minimum event 
duration required. In the case where a single scheduled event does not meet all of the user's total request, i.e. not enough time to downlink all data, additional UIS requests come from the user. Within the request protocol there is a minimum link time so that the Event Manager doesn't schedule events too short to be useful. Details about the UIS protocol are described in section II.

Conflict resolution between users is also a function of the Event Manager. Since there is only one SCaN Testbed, additional user spacecraft are simulated on the ground with separate computers running a version of the flight Service Manager. These simulated users can be configured to act as various mission types, some with periodic requests for service or more random, some with large science data collection rates or smaller rates. Currently a rulebased approach is used to resolve user conflicts for service, but there are machine learning techniques being explored to handle this function more optimally. A more intelligent Event Manager could also target anomaly detection in the system and/or individual user.

\section{E. System Operations}

The UIS experiment runs on SCaN Testbed in conjunction with the SN and GRC ground station link connectivity, as illustrated in Figure 9. Both the control plane signals for service requests/responses (green) and the data plane service links (blue) are shown in the figure. The TDRSS was used for both control and data plane links, while the GRC ground station was only used for scheduled service downlinks. The ground stations associated with the SN relay TDRS's are located at White Sands Complex (WSC) and the Guam ground terminal, which are also where the DAS receivers reside. There are terrestrial connections from and to WSC for the GRC mission operations center (MOC) that carry the received UIS requests, as well as the request responses from the Event Manager. For this experiment a scheduled multiple access forward link service was used to transmit the responses back to SCaN Testbed.

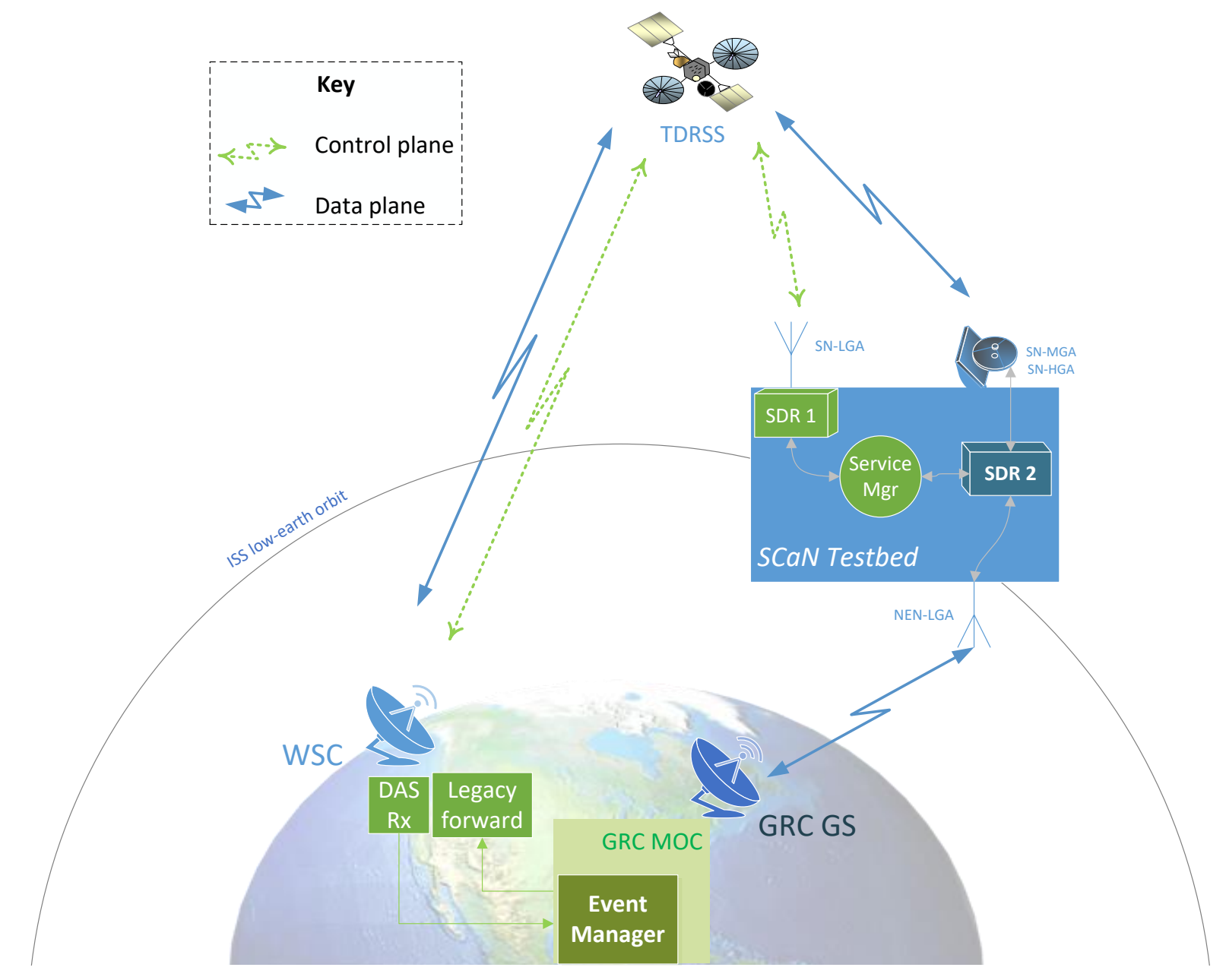

Figure 9. SCaN Testbed UIS Experiment System Configuration

American Institute of Aeronautics and Astronautics 


\section{Flight Testing and Results}

UIS testing using the SCaN Testbed took place on six separate days in August and September of 2017. Initially testing used only SNAS scheduled TDRSS time, and then later access times to the GRC ground station were added. Emulated science data was generated on-board SCaN Testbed continuously, triggering a request for service message from the Service Manager when the buffers reached a configurable fill level. During test days the "any TDRS" mode was setup with the SN's DAS service such that the UIS request transmission could be picked up anywhere in the ISS orbit. However, there were small gaps in coverage when the non-steerable antenna was extremely off-boresight with a TDRS or there was blockage from ISS structure (e.g. solar arrays). Receiving acknowledgements and access information from the ground Event Manager back to SCaN Testbed was more challenging.

The broadcast service of the $\mathrm{SN}$ is a future service of the Next Generation Architecture. ${ }^{6}$ To emulate the broadcast service the experiment employed a scheduled forward service with TDRSS to emulate the broadcast (see Figure 7). However, with other SN users in orbit, this service could not be scheduled continuously, resulting in several breaks in the UIS control plane path back to SCaN Testbed. The on-board Service Manager is configured to wait five minutes for a request acknowledgement, upon which time the request is resent with the assumption it was not received the first time. The service identification number in the message header allows the ground Event Manager to ignore multiple repeat requests during times when the control plane path back to SCaN Testbed was unavailable.

Whenever the forward link was available and the DAS link was steady, there was a quick turnaround from request to potential data plane access. Some requests resulted in high data rate access events only 15 minutes later. The primary turn-around limitation is the time needed to configure $\mathrm{SCaN}$ Testbed SDRs and the antenna pointing system, as well as time needed for TDRSS to slew an antenna toward the user location. As illustrated in Figure 10, these UIS operations are a dramatic improvement from SCaN Testbed's previously used three week inadvance forecast period requests. During the six days of the experiment a total of 17 requests were granted and executed, resulting in over $11 \mathrm{~GB}$ of data downlinked.

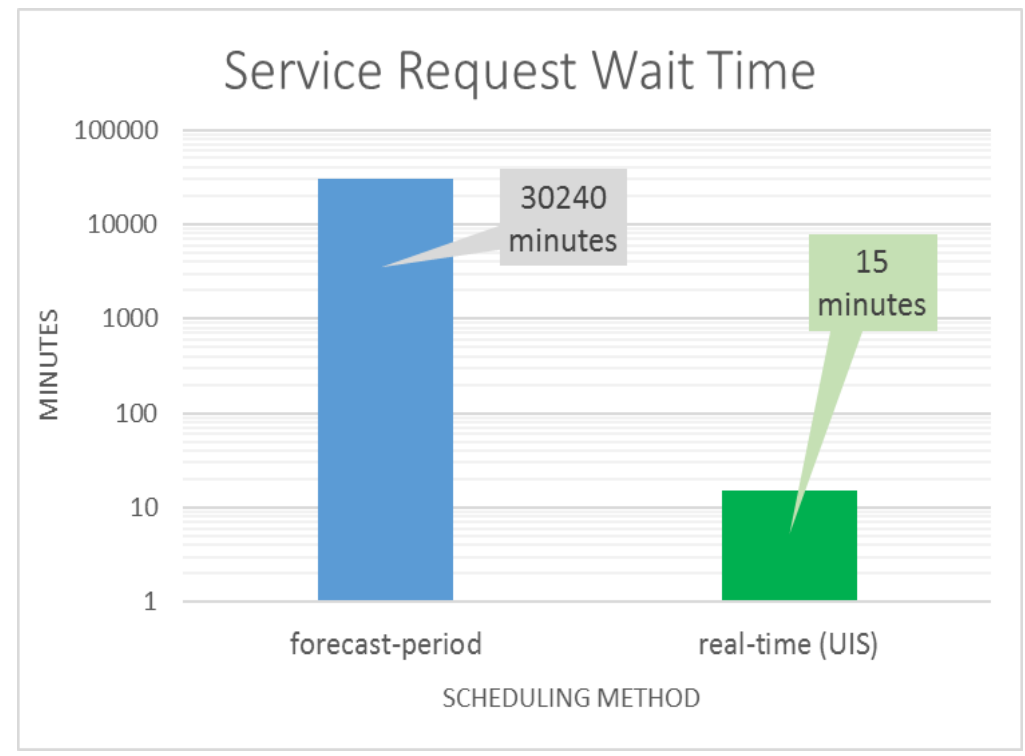

Figure 10. SCaN Testbed UIS Response Time versus Traditional Practice

\section{A. Single SDR Operations}

All user spacecraft may not have multiple radios so as to dedicate one solely to UIS control plane operations. Therefore, tests using just one SDR on-board SCaN Testbed for both control and data plane links were executed. Exploiting one of the key advantages of SDRs, reprogramability, allows one radio to perform different communication functions during different parts to the mission. For UIS the single SDR is primarily programmed with the control plane functions, which in the case of SCaN Testbed is the DAS transmit waveform and the emulated broadcast receive waveform. After a request has been made, acknowledged, and access information received and acknowledged, the SDR is reconfigured per the specific data plane access. On SCaN Testbed not only does the SDR's waveform need to be changed, but the antenna connection is re-routed from the low-gain nonsteerable to the medium steerable antenna, or to the earth-facing antenna if it is a ground station access. Once the downlink access is finished the SDR and antenna system is re-configured back to the control plane mode and starts listening for any "broadcasts." Three UIS requests were executed while in this single SDR operations mode, each transition from control to data plane and vice versa taking about one minute. 


\section{B. Automated Eclipse Tracking}

A unique opportunity presented itself in August with the North American solar eclipse. The path of the ISS passed through the moon's shadow, at least partially, for three orbits. The steerable antenna controlled by the orbit propagator software can autonomously track any celestial object. On August 21, 2017 the SCaN Testbed was sent access information via the UIS protocol to autonomously track the sun, instead of a TDRS. As the ISS moved in and out of the moon's shadow RF spectrum samples were collected with the Ka-band SDR. Over 2 gigabytes of raw ADC samples were collected during three orbits, some sampling in the eclipse zone and some full sun for comparison. This scenario is representative of a science mission collecting data from instruments.

\section{Future Work}

\section{A. Phase II testing with SCaN Testbed}

There are several dimensions of automated space communications towards which this work can be expanded. In the near future there are plans to overlay various networking applications on the UIS operations. These applications may include disruptive tolerant networking, secure transmissions, and cross-layer optimization. ${ }^{13}$ Adaptive waveforms previously run on SCaN Testbed can be integrated to UIS operations so that the maximum data rate is achieved, for example, based on the real-time link margin. ${ }^{14}$

Further benefits of optimization and adaptability may be incorporated into system automation by applying cognitive techniques. Network management may improve by learning user request patterns and specific relay satellite differences. For example, the Event Manager may be able to anticipate services requests with missions that have a regular cadence, and a spacecraft may learn how to optimize service request frequency and duration. Service Manager simulated users from this demonstration allow various cognitive scenarios and machine learning approaches to be explored.

\section{B. Commercial Services}

The TDRSS constellation will be replenished beginning in the 2030 time frame, as the satellites reach their design lifetime. NASA is considering the use of commercially provided RF and optical space-to-space SN services to augment any unique NASA owned and operated elements that remain. The dual use of NASA and commercial assets must be considered as the UIS architecture evolves.

Today the NEN uses a combination of both commercial and NASA-owned ground assets. Selection, use, and scheduling of these systems is controlled by NASA and occurs on a pre-planned basis determined by mission needs, station availability, and cost of use. However, employing commercially provided SN services is a significant cultural, technical, and enterprise shift in mission services and operations. The future NASA SN architecture is envisioned as a disaggregated set of providers and space elements providing a more scalable, extensible, and resilient network. Disaggregation allows the use of multiple independent providers to obtain both routine and critical services, while preventing dependence on any one supplier. Disaggregation of services allows placing capability where and when needed at lower cost than the multi-service per spacecraft approach used today. A tradeoff is underway of system complexity with user spacecraft burden, life-cycle costs and technical cost risks as the future architecture definition continues.

The UIS architecture described in this paper, is a key element of the future NASA space architecture. Simplifying the scheduling of spacecraft services among both NASA assets and potentially multiple commercial providers will require an automated process to improve the availability of resources to the missions and the efficient use of space and ground assets. Envision the UIS common scheduling system hosting both a network service and event manager extended to include not only NASA's space assets but also include other commercial assets available for NASA's use. With the disaggregation of services, the broadcast service and low rate demand access service could be provided by either a NASA asset or commercial service. The system could then schedule a data or other service from a NASA or commercial asset. Each assets location (orbital or ground), capability, capacity, and functionality would be known by the network service manager and allocated appropriately by the event manager. While more complex (although well within the functionality of the software) and taking into account the negotiated use allotted to each asset, the UIS allows better management of the data services, asset selection, satisfying mission needs, and accounting of service and use across the entire enterprise.

\section{Infusion to Operations}

Traditionally, mission users undergo extensive link testing during the integration lifecycle phase to ensure compatibility with the provider network prior to launch and operations. Similarly, operational infusion of control plane services such as UIS will require application-layer testing to ensure proper behaviors and control information 
flows between the user and network. While this experiment has demonstrated the feasibility and a small set of the potential benefits of UIS technology, further work is necessary to demonstrate the scalability of the technology for multiple hardware and software environments to facilitate operational infusion.

The growing prevalence of cubesats and other small satellite platforms present an ideal early adopter user set due to their generally higher technology risk tolerance and motivation to avoid costs through automation. In particular, it will be necessary to demonstrate that the UIS flight software can run effectively within the more limited processing and storage resource constraints of cubesat and small satellite platforms. Additionally, to facilitate wide scale adoption, the UIS flight software must be capable of running in multiple mission platform hardware and realtime software environments. Further work is needed to demonstrate UIS flight software encapsulation or refactoring into emerging flight software environments popular with cubesat and small satellite users, such as NASA's Core Flight Software. ${ }^{15}$

\section{Conclusion}

With ever increasing use of space for government and commercial purposes, changes to the supporting network of communications services are necessary. This paper has described one approach to provide users with more timely network access with User Initiated Services. This concept, including a draft UIS messaging protocol, was demonstrated using NASA's SCaN Testbed orbiting on the ISS. Over 200 minutes of network service was autonomously requested and utilized during the experiment, scheduling with as little as 15 minutes lead time instead of the traditional three weeks.

\section{Acknowledgments}

For implementing and executing this demonstration, the authors would like to thank the SCaN Testbed flight operations team and software developers, including Larry Vincent, Jason Murray, Gus Gemelas, Mike Evans, Dan McGiffin, Dave Brooks, Marie Piasecki, Beth Curtis, Dean Schrage, Al Rybar, and Wes Eddy. In addition, there is appreciation for contributors to this effort from Goddard Space Flight Center, namely Jacob Burke, Jacob Barnes, Mark Sinkiat; and Colorado State support from Thomas Bradly and John Borky.

\section{References}

\footnotetext{
${ }^{1}$ Sokol, Joshua. "Minisatellite surge spurs downlink infrastructure," Science Magizine, Vol. 357, Issue 6358, 29 September 2017.

${ }^{2}$ Israel, D., G. Heckler, and R. Menrad, Space Mobile Network: A Near Earth Communications and Navigation Architecture, presented at the IEEE Aerospace Conference, Big Sky, MT, United States, 2016.

${ }^{3}$ Goddard Space Flight Center. “CubeSat/Small Sat,” Technology Transfer, Vol 15, Number 3. Summer 2017.

4 Akaiwa, Yoshihiko. Introduction to Digital Mobile Communication. Wiley Series in Telecommunications and Signal Processing. May, 2015.

${ }^{5}$ Wertz, James and Wiley Larson (ed.). Space Mission Analysis and Design. Springer. 1999.

${ }^{6}$ Reinhart, Richard C.; Schier, James S.; Israel, David J.; Tai, Wallace; Liebrecht, Philip E.; Townes, Stephen A. "Enabling Future Science and Human Exploration with NASA's Next Generation near Earth and Deep Space Communications and Navigation Architecture", 68 ${ }^{\text {th }}$ International Astronautical Congress, September 2017.

${ }^{7}$ Roberts, Christopher, Robert Morgenstern, David Israel, John Borky, and Thomas Bradley. "Preliminary results from a modeldriven architecture methodology for development of an event-driven space communications service concept." Space-Terrestrial Internetworking Workshop, IEEE Wireless for Space and Extreme Environments, Montreal, Canada. October 2017.

${ }^{8}$ Haleplidis, E., K. Pentikousis, S. Denazis, et al., Software-Defined Networking (SDN): Layers and architecture Terminology, Internet Research Task Force (IRTF) Request for Comments: 7426, ISSN: 2070-1721, January 2015.

${ }^{9}$ Reinhart, Richard C.; Lux, James P., "Space-Based Reconfigurable Software Defined Radio Test Bed Aboard International Space Station”, AIAA Space Ops Conference, GRC-E-DAA-TN13717, May 2014.

${ }^{10}$ Chelmins, David; Mortensen, Dale; Shalkhauser, Mary Jo; Johnson, Sandra K.; Reinhart, Richard, "Lessons Learned in the First Year Operating Software Defined Radios in Space", AIAA International Communications Satellite Systems Conference; GRC-E-DAA-TN16240 August 2014.

11 Welch, B. W., Piasecki, M. T., and Schrage D. S., "Use of a Closed-Loop Tracking Algorithm for Orientation Bias Determination of an S-Band Ground Station", GRC-E-DAA-TN25134, 2015.

${ }^{12}$ Gitlin, Thomas A.; Kearns, Walter; Horne, William D., "The NASA space network demand access systems (DAS)", 2011.

${ }^{13}$ Clark, Gilbert, Wesley Eddy, James Barnes, Sandra Johnson, and David Brooks. "Architecture for Cognitive Networking within NASAs Future Space Communications Infrastructure", 22nd Ka and Broadband Communications Conference and the 34th AIAA International Communications Satellite Systems Conference, Cleveland, OH, 10/18/2016.
} 
${ }^{14}$ Downey, Joseph, Dale Mortensen, Michael Evans, Janette Briones, and Nicholas Tollis. "Adaptive Coding and Modulation Experiment with NASA's Space Communication and Navigation Testbed", 22nd Ka and Broadband Communications Conference / 34th AIAA International Communications Satellite Systems Conference, Cleveland, OH, 10/18/2016.

15 McComas, David; Wilmot, Jonathan; Cudmore, Alan; "The Core Flight System (cFS) Community: Providing Low Cost Solutions for Small Spacecraft”, AIAA/USU Conference on Small Satellites, SSC16-IV-1, GSFC-E-DAA-TN33786, August 2016. 